\title{
Chronic inflammation and frequent exacerbations in patients with COPD: the role of PDE4 inhibitors
}

Mitja Jevnikar

Chiara Torregiani

Department of Pneumology, University Hospital of Cattinara, Trieste, Italy

\author{
Address for correspondence: \\ Mitja Jevnikar \\ Department of Pneumology \\ University Hospital of Cattinara \\ Trieste, Italy \\ E-mail: mitja.jevnikar@hotmail.it
}

\section{Summary}

Inflammation represents an important hallmark of several diseases. In COPD the inflammatory lung response may be chronic and dysregulated with accumulation of inflammatory cells that causes lung parenchyma damage and airway remodeling. The inflammatory cascade involves different cells, mediators, proteins, oxygen species that leads the local inflammation to become systemic and promotes comorbidities and complications.

Different stimuli may worsen the COPD pathology, including the well known COPD exacerbation, a peak of local and systemic inflammation. Also hypoxia may stimulate inflammatory genes worsening local and systemic level of inflammation.

Understanding the inflammation can allow us to differentiate COPD phenotype and lead as to personalize the therapy. Several have the specific target of inflammation, particularly PDE4 inhibitors may have a role in certain COPD phenotype.

KEY WORDS: chronic obstructive pulmonary disease, COPD, inflammation, phosphodiesterase-4, roflumilast, acute exacerbation.

Dysregulated and prolonged inflammation as a pathologic process in COPD

Inflammation, a response triggered by damage to living tissues, represents the hallmark of several lung diseases. A correct inflammatory response is a defense mechanism to protect individuals from infections and injuries. Nevertheless, the inflammatory response may be dysregulated and prolonged becoming itself a cause of disease, as we can find in several chronic lung diseases. It is the case of chronic obstructive pulmonary disease (COPD) where the airways, in particular the peripheral ones, lung parenchyma and pulmonary blood vessels are common places of altered prolonged inflammatory response (1). Chronic inhalation of irritants leads to the recruitment and accumulation of inflammatory cells, such as neutrophils, T-lymphocytes (CD8+) and macrophages (2) have been demonstrated in airways of COPD patients and this producing as main effects airway remodeling and lung parenchyma destruction. Both innate immunity with neutrophils, macrophages, eosinophils, NK cells, gamma delta cells and dendritic cells and adaptive immunity with $T$ and $B$ cells have roles in COPD together with the activation of structural cells as epithelial cells, endothelial cells and fibroblasts (2).

Increased number of macrophages, have been found in COPD patients airways, up to 5-10 fold and the number of macrophages has been related to emphysema entity (2). Once activated by cigarette smoke, macrophages may orchestrate the chronic inflammatory response with release of both inflammatory mediators and chemokines that amplify inflammation, of reactive oxygen species, of elastolytic enzymes, of smooth muscle constrictors, of mucus gland activators and of matrix metalloprotease enzymes (MMPs). Neutrophils are the most represented cells, their number has been related to COPD severity, and they are found mostly in patients sputum and BAL probably because of their rapid transit and less in the airway walls. Smoking has direct stimulatory effect on granulocyte production, release from bone marrow and on their survival. Neutrophils are directed to airways because of several chemokines secreted by different cells as macropha-ges, $\mathrm{T}$ cells, epithelial cells and the same neutrophils activated in the airways of COPD patients. These cells are source of reactive oxygen metabolites, inflammatory cytokines, lipid mediators, antibacterial peptides and tissue damaging enzymes as serine proteases, cathepsine G, proteinase- 3 and matrix-metalloproteinase (MMP-8, MMP9). Neutrophil products also induce mucus hypersecretion by both an acute secretagogue effect and by augmentation of the bronchial mucus producing apparatus. Also lymphocytes, mostly CD8+ cell, eosinophils and mast cells found in both the large and small airway walls (2). 
All these cells cohoperate to the inflammatory cascade that triggers the release of inflammatory mediators such as tumor necrosis factor- $\alpha$ (TNF $\alpha$ ), interferon- $\gamma$ (IFN $\gamma$ ), interleukins (IL-1, IL-6, IL-8) and fibrinogen (3). These mediators sustain the inflammatory process and lead to tissue damage and different systemic effects.

The oxidative agents are implicated in both the generation of mucous metaplasia in chronic bronchitis and in the destruction of the lung tissue in emphysema. Local inflammation pathway is composed by reactive oxygen species (ROS), a decreased level of alpha 1 antitrypsin, increased $\mathrm{H} 2 \mathrm{O} 2$, NO and S-nitrosothiol level and several damages to lipid membrane of epithelial cells. ROS may cause injury to the epithelial and endothelial cells through membrane lipid peroxydation, mitochondrial damage, and endoplasmic reticulum stress resulting in unfolded protein response (UPR). Chronic inflammation leads to various structural changes in the lung, which further perpetuate airflow limitation (Figure 1). The disequilibrium between the oxidant agents and antioxidant agents leads to inflammation and causes the typical airway disease of COPD patients (4). In fact, inflammation of the airways and emphysema with alveolar attachments distruptions in small airways increase airway resistance, rise the flow obstruction and dynamic hyperinflation, increases the work of the diaphragm and all this factors producing the clinical symptoms of dyspnea.

\section{The role of PDE4}

The inflammatory cascade involves different messenger proteins that regulate the inflammatory process. In the lung, the second messenger cyclic AMP (cAMP) is involved in the regulation of inflammatory cells, mucociliary clearance and pulmonary vascular remodeling (6). It suppresses immune and inflammatory cell activity (in inflammatory cells such as neutrophils, T-lymphocytes and macrophages), relaxes airway smooth muscle and modulates pulmonary nerve activity (7). In turn, an elevated concentration of intracellular CAMP activates protein kinase A, which enhances phosphorylation of proteins may inhibit numerous inflammatory cell functions, e.g. proliferation and release of cytokines and chemokines, reactive oxygen species, arachidonic acid metabolites, chemotaxis and proteases. cAMP modulates human airway epithelial cells that might support mucociliary clearance, human lung fibroblasts to potentially prevent a fibrotic response and the prolifera-
The enzyme phosphodiesterase 4 (PDE4) degrades cAMP into its inactive form, thereby stimulating the activity of inflammatory cells. tion of pulmonary artery smooth muscle cells thereby reducing pulmonary vascular remodelling (8).

The enzyme phosphodiesterase 4 (PDE4) degrades cAMP into its inactive form, thereby stimulating the activity of inflammatory cells (Figure 2).

\section{The role of local inflammation in COPD}

The local inflammation of the lung generates a systemic inflammatory response that subsequently becomes chronic, leading to comorbidities and complications with an increase of cardiovascular risk, loss of muscle mass, decrease of bone density. An important effect of systemic inflammation is endothelial dysfunction. The main function of the endothelial cells is to maintain homeostasis of the vascular wall by adjusting the tone, permeability, adhesion of inflammatory cells, platelet aggregation and to prevent the activation of the coagulation cascade (Figure 3 ). Nitric oxide has a central role in endothelial function through the powerful vasodilatory, anti-inflammatory, anti-platelet and anti-proliferative effect, while to endothelin should be given the role of the most potent endogenous vasoconstrictor. An imbalance between these two factors with the possible in-

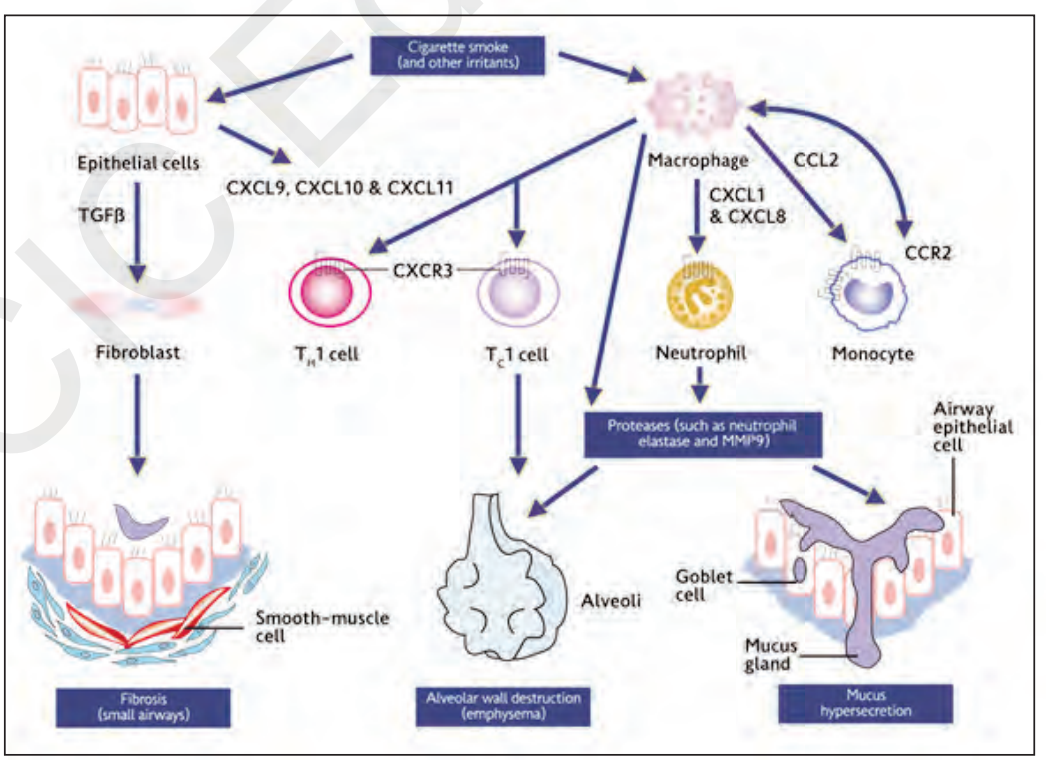

Figure 1 - Inflammatory and immune cells involved in COPD. 


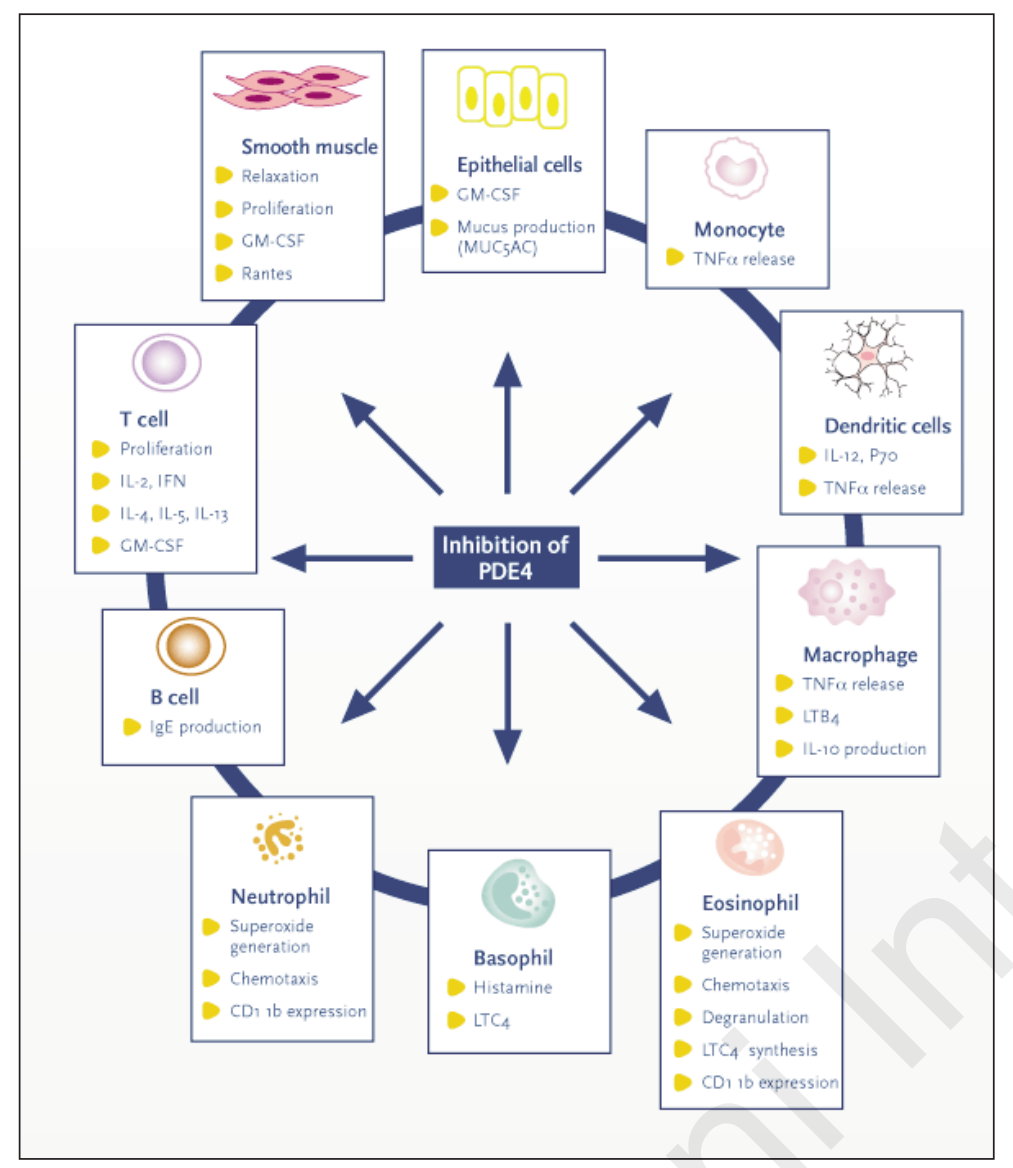

Figure 2 - Effect of PDE4 on inflammatory cells.

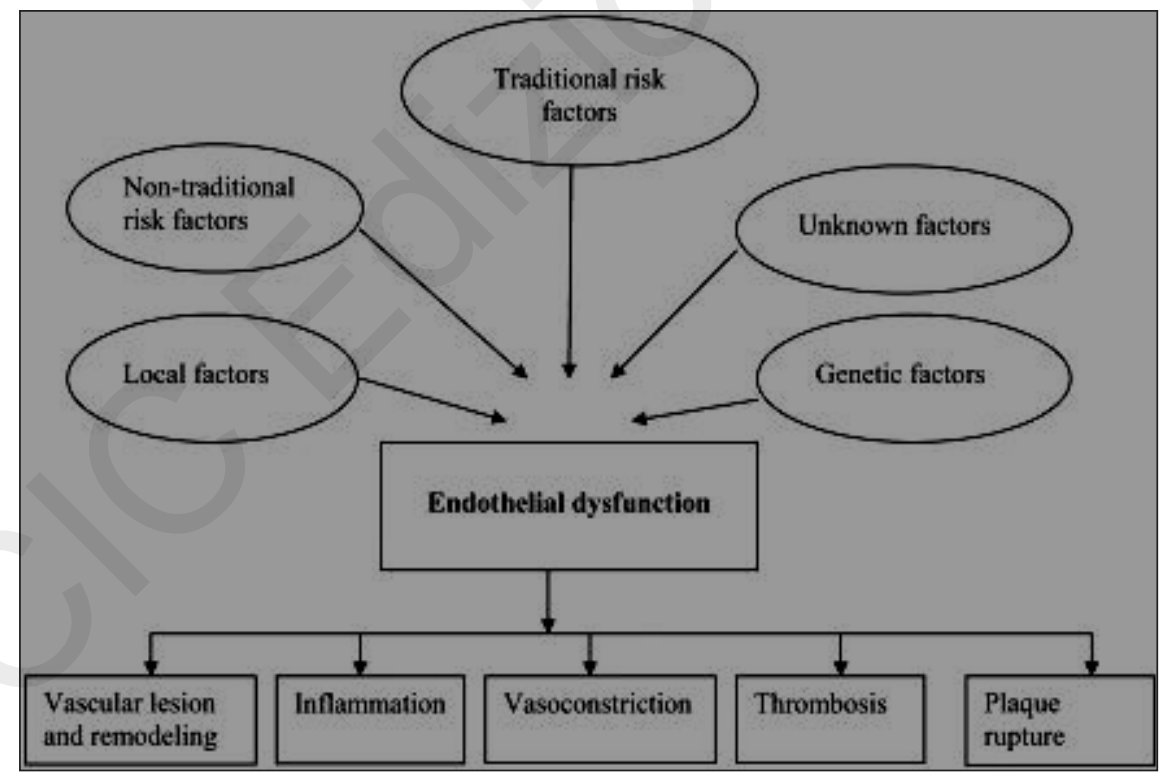

Figure 3 - Endothelial dysfunction in COPD.

volvement of other factors, determines the endothelial dysfunction (5). This condition is associated with atherosclerosis and several cardiovascular diseases such as hypertension, coronary syndromes, diabetes and chronic renal failure. A number of comorbidities can be explained with this mechanism. In fact to describe the comorbidities of COPD we could use the term "chronic inflammation". 


\section{The role of hypoxia}

Hypoxia is an important consequence of pulmonary disease. Results of chronic hypoxia are well known, but al-

A down-regulation by
hypoxia is seen in ge-
nes involved in cyto-
skeleton maintenan-
ce (Rho kinase),
mRNA processing
(heterogeneous nu-
clear ribonucleopro-
tein H1 and splicing
factor) and DNA re-
pair (REV3).
so the intermittent hypoxia has been recently shown to play an important role. In fact intermittent hypoxia reduces the resistance and endurance of the exercise, acts on systemic inflammation and on kidney function reducing renal blood flow and producing sodium retention (27). Based on studies of sleep apnea syndrome, the intermittent hypoxia shows an increase of two main factors: HIP and NF-kB (Figure 4). Hypoxia-inducible factor (HIF) -1 is a heterodimeric transcription factor, that can bind and regulate the expression of genes involved in metabolism (adenylate kinase 4, galactokinase) and apoptosis (galectin-3 and gelsolin). A down-regulation by hypoxia is seen in genes involved in cytoskeleton maintenance (Rho kinase), mRNA processing (heterogeneous nuclear ribonucleoprotein $\mathrm{H} 1$ and splicing factor) and DNA repair (REV3). All these genetic regulations cause a proinflammatory response induced by hypoxia and promote enhanced survival of myeloid inflammatory cells, such as granulocytes, monocytes and macrophages, resulting in their functional longevity and inflammatory response.

\section{Inflammation and acute exacerbations of COPD}

COPD exacerbations is defined generally as changes in respiratory symptoms. Focusing on inflammation, COPD exacerbation could be defined as a peak of inflammation during the course of the disease. Serum biomarkers such as various cytokines, adipokines, C-reactive protein and coagulation factors are elevated during exacerbations.

An increase of lung inflammation, as during the exacerbation, worsens the systemic

An increase of lung inflammation, as during the exacerbations, worsens the systemic inflammation that leads to an increase in major acute events, especially cardiovascular events.

inflammation that leads to an increase in major acute events, especially cardiovascular events. The same happens in pneumonia in the post-acute period (9). Exacerbations also are related to means functional decline, symptoms increase, quality of life worsening, increased risk of hospitalization and mortality, and exposes the patient him/herself to relapsing further exacerbations. The prognosis becomes unfavorable in most patients who have exacerbations especially if they require hospitalization. The detection of higher blood biomarkers during exacerbation compared with baseline, such as CRP, IL-8, TNF- $\alpha$, leptin, endothelin-1, eosinophil cationic protein, myeloperoxidase, fibrinogen, IL-6, $\alpha 1$-antitrypsin, leukotrienes E4 and B4 (Table 1) confirm that exacerbation deals with a systemic peak of inflammation.

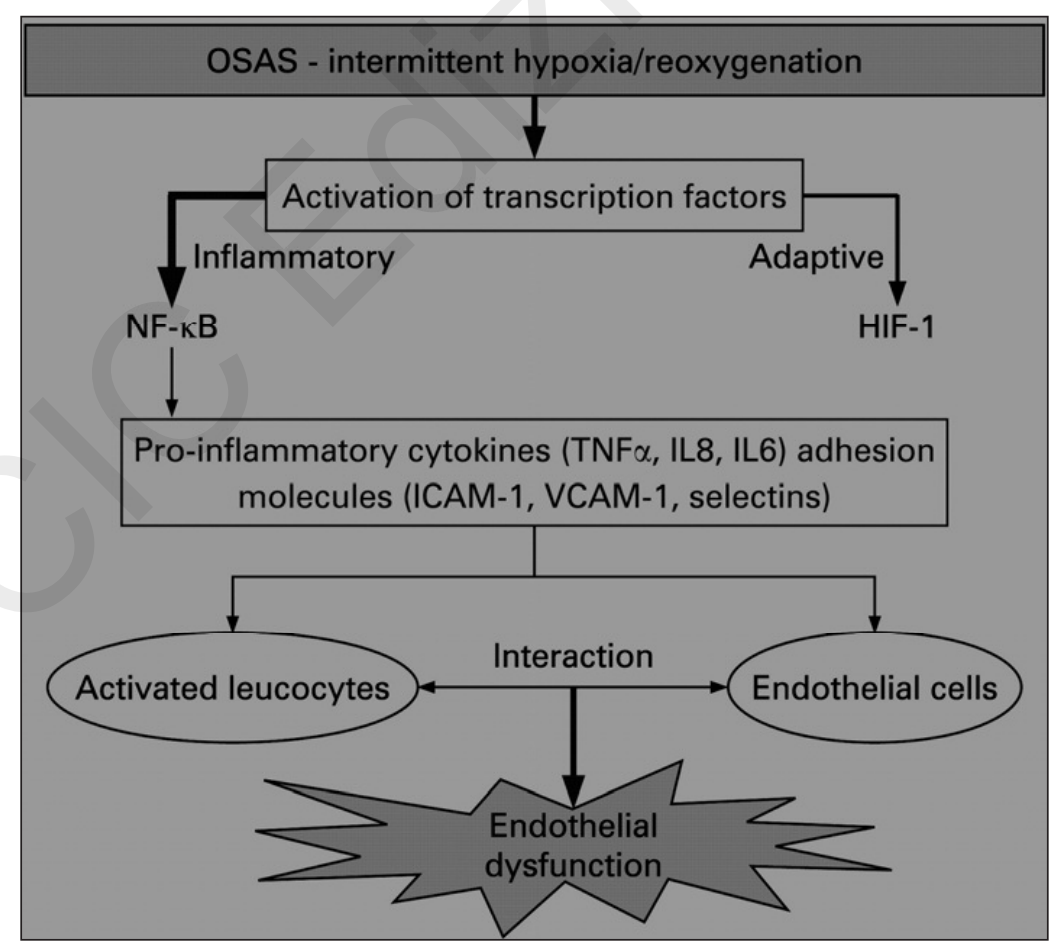

Figure 4 - Intermittent hypoxia and inflammation. 
TABLE 1 - Systemic mediators known to be elevated during chronic obstructive pulmonary disease exacerbations.

C-reactive peptide
Copeptin
IL-8
IL-6
Tumor necrosis factor- $\alpha$
Leptin
Eosinophillic cationic protein
Myeloperoxidase
$\alpha 1$-Antitrypsin
Leukotrienes E4 and B4
Fibrinogen
Myeloid progenitor inhibitory factor-1 (MPIF-1)
Pulmonary and activation-regulated chemokine (PARC)
Soluble intercellular adhesion molecule-1 (sICAM-1)
Adiponectin (ACRP-30)

All these molecules bind and trigger the activation of vascular endothelium on luminal surface of the vessels and promote endothelial dysfunction and atherosclerosis (10). This leads to a destabilization of atherosclerotic plaque and cardiovascular complications.

Because of the pivotal role of inflammation in the genesis of systemic comorbidities we should act on it and particularly on exacerbations. Several studies have been done to find solutions to improve the outcomes. The bronchial epithelium and endothelium are a target reached by inhalation therapy. The bronchodilators have an important role in exacerbations prevention as the fixed combination $\alpha-2$ agonists and steroids (TORCH study), the tiotropium (UPLIFT study), the fixed combination with tiotropium (INSPIRE study). On inflammation is also acting the inhibitor of phosphodiesterase-4 Roflumilast, an available drug which acts either on CD8 cells, macrophages, neutrophils, epithelial cells and smooth muscle. The mechanism of this drug is to prevent the conversion of 3'-5 'cyclic AMP to AMP. It increases the amount of 3'-5' cyclic AMP in the cell and modulates the phosphorylation of the inflammatory proteins (Figure 5).

The rationale for PDE4 inhibitors in COPD patients with chronic inflammation

Differently from asthma, inhaled corticosteroids (ICS) shows a limited response in COPD (11). The low number of glucocorticoids receptors (GR) in neutrophils may explain, in part, why inflammation in COPD is insensitive to glucocorticoids as monotherapy. Furthermore, COPD inflammation is different from asthma one making steroid-resistance much more frequent (12). The reduced effect of ICS in COPD seems due to the marked reduction in histone deacetylase 2 (HDAC2), the nuclear enzyme that corticosteroids require to switch off ac-

tivated inflammatory genes (13), rendering these patients resistant to the effects of ICS. The reduction in HDAC2 is thought to be secondary to oxidative stress, both independent and by activation of phosphoinositide3-kinase- $\delta$ (PI3K $\delta)$. Inhibition of PI3K $\delta$ has recently shown to restore corticosteroid sensitivity in mice and may hold therapeutic promise $(14,15)$. Marwick et al. (16) have shown that formoterol reverses oxidative stress-induced

A large part of pre-clinical research has shown that PDE4 inhibition has the potential to target the three main components of COPD: bronchoconstriction, mucus hypersecretion and airway remodeling.

corticosteroid insensitivity via PI3Kס. Low-dose theophylline has shown to enhance the antiinflammatory effects of steroids during exacerbations of COPD (17) and seems to have the capacity to restore the reduced HDAC2 activity in COPD macrophages (18). Roflumilast has also been shown to increase the ability of formoterol to enhance glucocorticoid-dependent gene transcription in human airway epithelial cells (19). A large part of pre-clinical research has shown that PDE4 inhibition has the potential to target the three main components of COPD: bronchoconstriction, mucus hypersecretion and airway remodelling. As PDE4 is the major cAMP-metabolizing enzyme, the inhibition of PDE4 suppresses the inflammatory response and the epidermal growth factor receptor-induced Mucin 5AC over-expression, that directly inhibits mucus production. Inhibition of PDE4 may also lead to minimization of airway remodeling by suppressing the release of TNF- $\alpha$ (20).

The PDE4 inhibitor Roflumilast is now indicated for maintenance treatment of severe COPD (FEV 1 post-bronchodilator less than $50 \%$ predicted) associated with chronic bronchitis in adult patients with a history of frequent exacerbations as add on to bronchodilator treatment. In this patient population, Roflumilast $500 \mu$ g oncedaily may reduce exacerbation rate and improves lung function.

\section{Are all the COPD a same phenotype based on inflam- mation?}

Several guidelines define COPD as an inflammatory disease, but there are few or no specific recommendations on the assessment and targets for reduction in inflammation, as these are not established (2123). Nevertheless, there is for sure a COPD phenotype where the inflammation is predominant and a potential target of therapy (24). A phenotype which needs more attention for its burden and that represents a population that completely cross the GOLD classification is the so-called "fre-

A phenotype which needs more attention for its burden and that represents a population that completely cross the GOLD classification is the so-called "frequent exacerbator" of the ECLIPSE study. quent exacerbator" of the ECLIPSE study (25). These patients, beyond exacerbations, have a more pronounced in- 


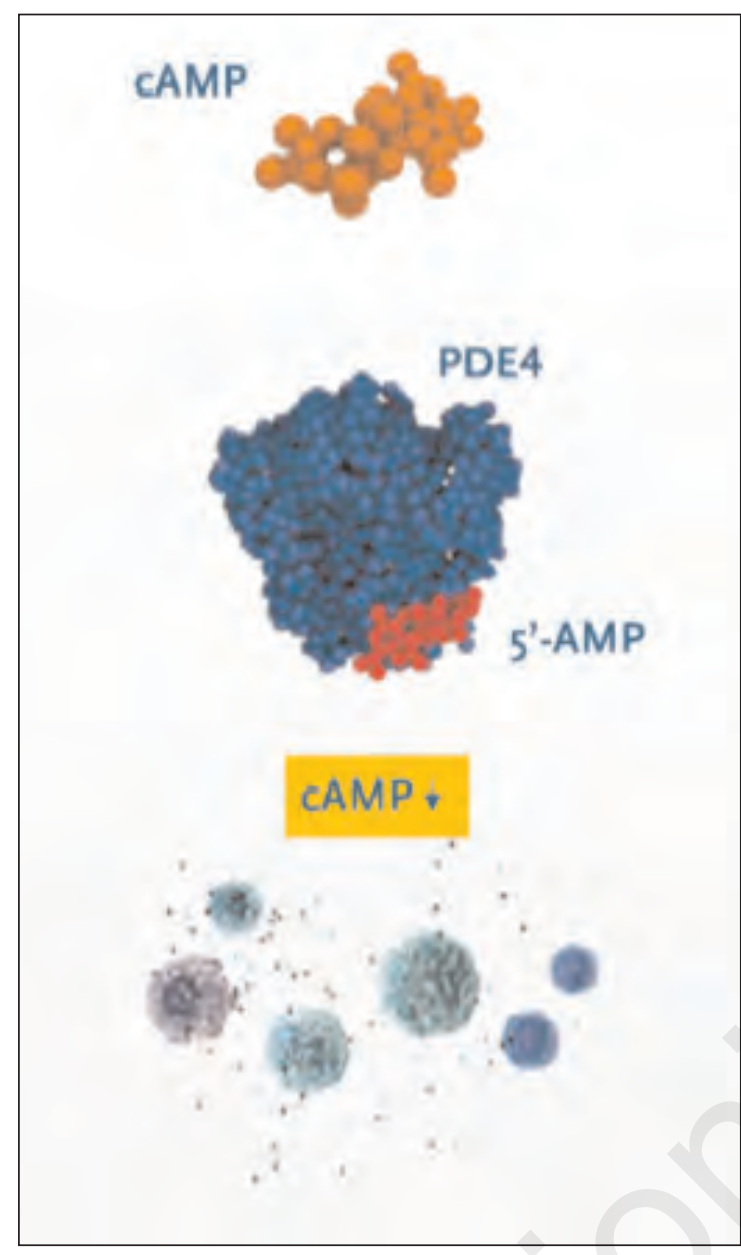

Figure 5 - Effect of PDE4 on cAMP.

flammation with higher levels of sputum interleukin IL-6 and IL-8 even in the stable state (26). We have to take in account this population and identify them, because of the worst prognosis and quality of life. The aim of future investigations will be the characterization of COPD phenotypes physiopathologically and therapeutically for a better prognostic assessment and therapeutic management $(24,27)$.

\section{References}

1. Hogg JC, Chu F, Utokaparch S, et al. The nature of small-airway obstruction in chronic obstructive pulmonary disease. N Engl J Med. 2004;350:2645-53.

2. Barnes PJ. Immunology of asthma and chronic obstructive pulmonary disease. Nat Rev Immunol. 2008;8:183-92.

3. Boots AW, Haenen GRMM, Bast A. Oxidant metabolism in chronic obstructive pulmonary disease. Eur Respir J Suppl. 2003 Nov.

4. MacNee W. Chest. Oxidants/antioxidants and COPD.2000 May;117(5 Suppl 1):303S-17S.

5. Endemann DH, Schiffrin EL. Endothelial dysfunction. J Am Soc Nephrol. 2004 Aug;15(8):1983-92.
6. Hatzelmann A, Morcillo E, Lungarella G, et al. The preclinical pharmacology of roflumilast - A selective, oral phosphodiesterase 4 inhibitor in development for chronic obstructive pulmonary disease. Pulm Pharmcol Ther. 2010:23;235-56.

7. Soto FJ, Hanania NA. Selective phosphodiesterase4 inhibitors in chronic obstructive lung disease. Curr Opin Pulm Med. 2005;11:129-34.

8. Grootendorst DC, Gauw SA, Verhoosel RM, et al. Reduction in sputum neutrophils and eosinophil numbers by the PDE4 inhibitor roflumilast in patients with COPD. Thorax. 2007;62;1081-7.

9. Corrales-Medina VF, Musher DM, Shachkina S, Chirinos JA. Acute pneumonia and the cardiovascular system. Lancet. 2013;381:496-505.

10. Libby P, Ridker PM, Maseri A. Inflammation and Atherosclerosis. Circulation. 2002;105:1135-1143.

11. Burge PS, Calverley PMA, Jones PW, et al. Randomised, double-blind, placebo controlled study of fluticasone propionate in patients with moderate to severe chronic obstructive pulmonary disease, the ISOLDE trial. BMJ. 2000;320:1297-303.

12. Schleimer RP. Effects of glucocorticosteroids on inflammatory cells relevant to their therapeutic applications in asthma. Am Rev Respir Dis. 1990;141:S59-S69.

13. Ito $\mathrm{K}$, Ito $\mathrm{M}$, Elliott WM, et al. Decreased histone deacetylase activity in chronic obstructive pulmonary disease. N Engl J Med. 2005;352:1967-197.

14. Ito K, Caramori G, Adcock IM. Therapeutic potential of phosphatidylinositol 3-kinase inhibitors in inflammatory respiratory disease. J Pharmacol Exp Ther. 2007;321:1-8.

15. Barnes P. New anti-inflammatory targets in chronic obstructive pulmonary disease. Nature Rev Drug Disc. 2013;12:543-59.

16. Marwick JA, Caramori G, Stevenson CS, et al. Inhibition of PI3Kdelta restores glucocorticoid function in smoking-induced airway inflammation in mice. Am J Respir Crit Care Med. 2009;179:542-548.

17. Cosio BG, Iglesias A, Rios AA, et al. Low-dose theophylline enhances the anti-inflammatory effects of steroids during exacerbations of COPD. Thorax. 2009;64:424-429.

18. Cosio BG, Tsaprouni L, Ito $\mathrm{K}$, et al. Theophylline restores histone deacetylase activity and steroid responses in COPD macrophages; J Exp Med. 2004;200 689-695.

19. Moodley T, Wilson SM, Joshi T, et al. Phosphodiesterase 4 inhibitors augment the ability of formoterol to enhance glucocorticoid-dependent gene transcription in human airway epithelial cells: a novel mechanism for the clinical efficacy of roflumilast in severe chronic obstructive pulmonary disease. Mol Pharmacol. 2013;83:894-906.

20. Giembycz MA, Newton R. How Phosphodiesterase 4 Inhibitors Work in Patients with Chronic Obstructive Pulmonary Disease of the Severe, Bronchitic, Frequent Exacerbator Phenotype; Clin Chest Med. 2014;35:203-217.

21. Global Initiative for Chronic Obstructive Lung Disease (GOLD). Global Strategy for the diagnosis, management,and prevention of chronic obstructive pul- 
monary disease(rev.2014). Available at: http://www. goldcopd.org/uploads/users/files/GOLD_Report 2014_Jun11.pdf. Accessed August 22, 2014.

22. Bellamy D, Bouchard J, Henrichsen S, et al. International Primary Care Respiratory Group (IPCRG) Guidelines: management of chronic obstructive pulmonary disease (COPD). Prim Care Respir J. 2006;15.

23. Qaseem A, Wilt TJ, Weinberger SE, et al. Diagnosis and management of stable chronic obstructive pulmonary disease: a clinical guideline update from the American College od Physicians, American College of Chest Physicians, American Thoracic Society, and European Respiratory Society. Ann Intern Med.
2011;155:179-91.

24. Sethi S, Mahler DA, Marcus $P$, et al. Inflammation in COPD: implications for management. Am J Med. 2012;125:1162-70.

25. Hurst JR, Vestbo J, Anzueto A, et al. Susceptibility to exacerbation in chronic obstructive pulmonary disease. N Engl J Med. 2010;363:1128-38.

26. Wedzicha JA, Brill SE, Allinson JP, Donaldson GC. Mechanisms and impact of the frequent exacerbator phenotype in chronic obstructive pulmonary disease. BMC Medicine. 2013;11:181.

27. Barnes PJ. Cellular and molecular mechanisms of chronic obstructive pulmonary disease. Clin Chest Med. 2014;35:71-86. 